\title{
N-acetyl Cysteine Therapy as Adjunctive Therapy for Treatment of Acute Hepatitis A
}

\author{
Mariam Abdulrhman Al-Fadhli', Mohammad Ahmed Saraya ${ }^{2}$, \\ Jafar Abdulrida Qasem ${ }^{3}$ \\ ${ }^{I}$ Department of Medicine, Infectious Disease Hospital, Ministry of Health, Kuwait. \\ ${ }^{2}$ Department of Tropical Medicine, Faculty of Medicine, Zagazig University, Zagazig, Egypt. \\ ${ }^{3}$ Department of Applied Medical Sciences, College of Health Sciences, Public authority for \\ Applied Education and Training, Kuwait.
}

Corresponding Author M A Saraya

Mobile: +96555499517

E mail: mdsaraya@yahoo.com

Key words: Hepatitis $A ; \mathrm{N}$-acetyl cysteine
Background and study aim: Hepatitis A is an acute, usually mild and self-limiting disease affecting the liver. We aim to assess the effect of oral $\mathrm{N}$-acetyl cysteine compared with placebo on length of hospital stay in adult patients who were admitted to the hospital with acute hepatitis A which might cause earlier resolution of hepatitis.

Subjects and Methods: 40 patients were diagnosed as acute hepatitis $\mathrm{A}$ and classified into two groups, the first one involved 20 patients who received oral $\mathrm{N}$ acetyl cysteine and supportive treatment, and the second one involved also 20 patients but they received placebo and supportive treatment. We measured complete blood count (CBC), kidney profile (KP), liver function test (LFT), blood glucose, C-reactive protein (CRP) and coagulation profiles on the day of presentation, and every other day till the

\section{INTRODUCTION}

Hepatitis A is an acute, self-limiting disease affecting the liver caused by hepatitis A virus (HAV) [1]. The disease varies in clinical severity from a mild illness lasting 1-2 weeks to a severely disabling disease lasting several months. Most patients, who are infected, recover completely with no permanent liver damage [2]. Acute hepatitis A does not become chronic and there is no chronic carrier state. On rare occasions the disease may be very severe, with fulminant hepatitis, hepatic coma and death [2]. Severity of illness is strongly age dependent; adult who are infected with acute hepatitis A tend to experience a much more severe form of the disease, whereas young children typically have a milder form of the disease, usually day of discharge from the hospital. Serological tests were done for HAV Immunoglobulin M (IgM), HEV IgM, HBsAg, HBcIgM, antibody to Hepatitis C virus.

Results: The mean length of hospital stay in the NAC group was 13.2 days compared with 14.3 days in the placebo group. Length of hospital stay differed significantly between groups. The mean time of reliving symptoms at presentation was 3.6 days in the NAC group and 4.4 days in the placebo group. The mean time of reliving symptoms at presentation was significantly lower in NAC group than in placebo group.

Conclusion: use of oral NAC as adjunctive therapy for treatment of acute hepatitis A was safe in these patients and was associated with a shorter length of patient stay in the hospital.

lasting from 1-3 weeks [3]. For adults over 50 years of age, case fatality can reach $2 \%$. The increased risk of death from fulminant hepatitis A can occur in persons with pre-existing chronic liver diseases. Infection with HAV confers life-long immunity [3].

Globally, hepatitis A is more common in regions of the world drinking contaminated water and with poor sanitation [4] and around 1.4 million symptomatic cases occur each year [2, 5].The adulthood are immune in the developing world because about $90 \%$ of children have been infected by age 10 [4]. Outbreaks occur in developed countries where vaccination is not widespread and children are not exposed to the infections when young [4]. In 2010, acute hepatitis A caused 102,000 deaths [6]. 
$\mathrm{N}$-acetyl cysteine (NAC) can be used as an antidote in paracetamol intoxication and also can be used as a mucolytic $[7,8]$. N-acetyl cysteine can increase the amount of glutathione within the cell and maintain cell integrity [9]. The necessity for a medicine to decrease the duration of acute viral hepatitis is obvious, but it has not been found yet. This problem might be solved with $\mathrm{N}$ acetyl cysteine, which protects the liver cells architecture by increasing the amount of glutathione within liver cells that reacts with reactive oxygen species (ROS) [10]. NAC was licensed for use in 1968 [11]. It is on the World Health Organization's List of Essential Medicines, it is not very expensive drug, safe and most effective medicines needed in a health system [12].

The objective of this study was to assess the effect of oral $\mathrm{N}$-acetyl cysteine compared with placebo on length of hospital stay in adult patients who were admitted to the hospital with acute hepatitis A which might cause earlier resolution of hepatitis.

\section{SUBJECTS AND METHODS}

This study was conducted between February 2014 and July 2015, at the Infectious Disease Hospital (IDH). The patients included in this study were diagnosed as acute hepatitis A. Diagnosis of acute hepatitis A was clinically based on the presence of symptoms, e.g. anorexia, nausea, vomiting, abdominal discomfort, fever, fatigue and jaundice and confirmed serologically by positive of HAV Immunoglobulin M (IgM) antibodies, indicating acute disease.

40 patients were confirmed diagnosis of acute hepatitis A and classified into two groups, the first one involved 20 patients who received oral $\mathrm{N}$-acetyl cysteine and supportive treatment, and the second one involved also 20 patients but they received placebo and supportive treatment. All patients were subjected to history taking and thorough clinical examination. We measured complete blood count (CBC), kidney profile (KP), liver function test (LFT), blood glucose, Creactive protein (CRP) and coagulation profiles on the day of presentation, and every other day till the day of discharge from the hospital. Also, all patients were investigated for HEV IgM, hepatitis $\mathrm{C}$ virus antibody, $\mathrm{HBsAg}, \mathrm{HBcIgM}$, and hepatitis delta virus antibody to exclude other causes of acute viral hepatitis.
Patients in the first group (NAC group) were given $600 \mathrm{mg} \mathrm{NAC}$ effervescent tablet orally once daily (600 mg/day), NAC was continued as long as required for normalization of laboratory investigations and the patients in the second group were given placebo orally 3 times a day (placebo group). All patients received supportive treatment, according to individual needs.

\section{Statistical Analysis:}

The statistical package for social sciences (SPSS) version 8.0 software was used for analysis the data. The t-test was used to evaluate the significance of differences between mean values of the study variables. The significance of differences between proportions was performed using the Chi-square test. Significant differences were expressed at $\mathrm{P}<0.05$.

\section{RESULTS}

Forty patients were included in the study, they were classified into two groups, $\mathrm{N}$-acetyl cysteine group and placebo group, each of them involved 20 male patients varying in age from 14 years to 29 years. Of these, 11 patients were Indian, 10 Egyptian, 2 Indonesian, 8 Syrian, and 9 Kuwaiti. Symptoms at presentation included fever, anorexia, nausea, vomiting, abdominal discomfort, fatigue, dark urine and jaundice.

At the time of admission, no significant differences were noted between the NAC group and the placebo group as regard to liver enzymes, total bilirubin, direct bilirubin, INR, platelets, white blood cells, C-reactive protein, and serum creatinine (Table I).

After 5 days of admission, we noted a significant decline in liver enzymes (ALT \& AST) and total bilirubin in the NAC group than the placebo group (table II). At time of discharge, no significant differences were observed between the two groups regarding total bilirubin, direct bilirubin, liver enzymes, INR, platelets, white blood cells, C-reactive protein, and serum creatinine (Table III).

The mean length of patient stay in the hospital in the NAC group was 13.2 days $( \pm 0.67)$ compared with 14.3 days $( \pm 0.75)$ in the placebo group. Length of hospital stay differed significantly between groups $(\mathrm{p}$-value $=0.03$, table III). All patients were started the treatment within one hour of admission to hospital. The mean time of reliving symptoms at presentation was 3.6 days 
in the NAC group and 4.4 days in the placebo group. The mean time of reliving symptoms at presentation was significantly lower in NAC group than in the placebo group (p-value $=0.05$, Table II).

Table I : Comparison between studied groups at time of admission

\begin{tabular}{|l|c|c|c|}
\hline \multirow{2}{*}{ Age } & \multicolumn{3}{|c|}{ On admission } \\
\cline { 2 - 4 } & NAC group & Placebo group & P-value \\
\hline ALT(U/L) & $18.1 \pm 4.6$ & $17.5 \pm 4.1$ & 0.6 \\
\hline AST(U/L) & $2574.21 \pm 157.2$ & $2496.7 \pm 149.3$ & 0.4 \\
\hline Total bilirubin $(\boldsymbol{\mu m o l} / \mathbf{L})$ & $1865.4 \pm 103.8$ & $1879.1 \pm 113.7$ & 0.83 \\
\hline Direct bilirubin $(\boldsymbol{\mu m o l} / \mathbf{L})$ & $34.5 \pm 4.36$ & $36.1 \pm 3.04$ & 0.23 \\
\hline Albumin $(\mathbf{g} / \mathbf{L})$ & $18.7 \pm 3.4$ & $19.3 \pm 3.6$ & 0.12 \\
\hline CRP & $38.5 \pm 5.4$ & $38.8 \pm 4.2$ & 0.85 \\
\hline INR & $13.04 \pm 3.03$ & $13.05 \pm 3.06$ & 1.0 \\
\hline Platelet & $1.43 \pm 0.3$ & $1.33 \pm 0.4$ & 0.78 \\
\hline WBCs & $171.9 \pm 4.3$ & $173.5 \pm 4.1$ & 0.94 \\
\hline S. creatinine $(\boldsymbol{\mu m o l} / \mathbf{L})$ & $6.7 \pm 1.3$ & $6.34 \pm 1.4$ & 0.83 \\
\hline
\end{tabular}

NAC $=$ N-acetyl cysteine; ALT $=$ Alanine transaminase; AST $=$ Aspartate aminotransferase; $\mathrm{CRP}=\mathrm{C}$-reactive protein; $\mathrm{INR}=$ international normalized ratio; $\mathrm{WBC}=$ White blood cells; $\mathrm{S}$. creatinine $=$ Serum creatinine .

Table II : Comparison between studied groups after 5 days from admission

\begin{tabular}{|l|c|c|c|}
\hline \multirow{2}{*}{ ALT } & \multicolumn{3}{|c|}{ After 5 days from admission } \\
\cline { 2 - 4 } & NAC group & Placebo group & P-value \\
\hline AST & $1057.1 \pm 78.2$ & $1409.3 \pm 86.2$ & 0.05 \\
\hline Total bilirubin $(\boldsymbol{\mu m o l} / \mathbf{L})$ & $503.2 \pm 60.1$ & $851.4 \pm 49.3$ & 0.04 \\
\hline Direct bilirubin $(\boldsymbol{\mu m o l} / \mathbf{L})$ & $24.3 \pm 3.4$ & $28.2 \pm 3.1$ & 0.04 \\
\hline Albumin $\mathbf{( g / L )}$ & $11.5 \pm 2.5$ & $13.3 \pm 2.4$ & 0.1 \\
\hline CRP & $35.1 \pm 4.4$ & $35.9 \pm 4.2$ & 0.24 \\
\hline INR & $9.0 \pm 3.2$ & $8.0 \pm 3.4$ & 0.62 \\
\hline Platelet & $1.23 \pm 0.2$ & $1.26 \pm 0.4$ & 0.81 \\
\hline The mean time of reliving symptoms (day) & $184.5 \pm 26$ & $183.7 \pm 23$ & 0.86 \\
\hline
\end{tabular}

$\mathrm{NAC}=\mathrm{N}$-acetyl cysteine; $\mathrm{ALT}=$ Alanine transaminase; $\mathrm{AST}=$ Aspartate aminotransferase $; \mathrm{CRP}=\mathrm{C}$-reactive protein; $\mathrm{INR}=$ international normalized ratio; $\mathrm{WBC}=$ White blood cells; $\mathrm{S}$. creatinine $=$ Serum creatinine . 
Table III: Comparison between studied groups at time of discharge

\begin{tabular}{|l|c|c|c|}
\hline \multirow{2}{*}{ ALT } & \multicolumn{3}{|c|}{ On discharge } \\
\cline { 2 - 4 } & NAC group & Placebo group & P-value \\
\hline AST & $250.35 \pm 21.97$ & $251.90 \pm 26.86$ & 0.92 \\
\hline Total bilirubin $(\boldsymbol{\mu m o l} / \mathbf{L})$ & $134.5 \pm 12.4$ & $139.9 \pm 16.8$ & 0.8 \\
\hline Direct bilirubin $(\boldsymbol{\mu m o l} / \mathbf{L})$ & $17.3 \pm 2.1$ & $18.1 \pm 2.2$ & 0.12 \\
\hline Albumin $(\mathbf{g} / \mathbf{L})$ & $7.5 \pm 1.3$ & $8.3 \pm 1.4$ & 0.61 \\
\hline CRP & $36.2 \pm 4.1$ & $35.7 \pm 4.1$ & 0.65 \\
\hline INR & $4.7 \pm 1.5$ & $4.75 \pm 1.3$ & 0.93 \\
\hline Platelet & $0.96 \pm 0.2$ & $1.08 \pm 0.24$ & 0.29 \\
\hline WBCs & $191.74 \pm 18.5$ & $189.0 \pm 20.6$ & 0.53 \\
\hline S. creatinine & $8.44 \pm 1.76$ & $8.41 \pm 1.81$ & 0.93 \\
\hline Length of stay (day) & $84.3 \pm 11.4$ & $85.5 \pm 11.78$ & 0.74 \\
\hline
\end{tabular}

$\mathrm{NAC}=\mathrm{N}$-acetyl cysteine; ALT $=$ Alanine transaminase; AST $=$ Aspartate aminotransferase $; \mathrm{CRP}=\mathrm{C}$-reactive protein; INR= international normalized ratio; $\mathrm{WBC}=$ White blood cells; $\mathrm{S}$. creatinine $=$ Serum creatinine.

\section{DISCUSSION}

NAC is a specially modified form of the dietary amino acid cysteine. When taken orally, NAC is thought to help the body make the important antioxidant enzyme glutathione. It has shown promise for a number of conditions, particularly chronic bronchitis $[\mathbf{1 3}, \mathbf{1 4}]$.

NAC has been proposed as supportive therapy for HIV. Despite some intriguing results, overall the evidence is inconsistent at best $[15,16,17]$. Recently, some studies have revealed good results and absence of side effects in chronic hepatitis $\mathrm{C}$ and hepatitis B patients who treated with NAC [18, 19, 20].

The complications due to acute viral hepatitis were considered to be more frequent in adults than in the pediatrics [20]. Recently, reports from hepatic transplant centers suggest that $26 \%$ of cases with acute liver failure are caused with acute hepatitis A [21]. Hepatitis A is a common infection in the world [22].

When symptoms of acute hepatitis A (AHA) occur, recovery from these symptoms may take several weeks or months. In this study, we noted the mean time of reliving symptoms at presentation was significantly lower in the NAC group than in placebo group. In the first 5 days after admission, we noted that the NAC group showed a much more rapid improvement in liver enzymes and total bilirubin than the placebo group. Also, in this trial, we noted an overall reduction in mean length of hospital stay of more than one day in patients with AHA who were given NAC compared with who were given placebo. These findings support our hypothesis that adjunctive treatment of AHA with oral NAC might change the immune response and thereby reduce morbidity and length of patients stay in hospital.

The optimal duration of administration of NAC is not well known up tell now [23]. In this study, the duration of administration of NAC was between 11 days and 14 days, without observation any undesirable side effects, e.g. nausea, vomiting, itching, rash, hypotension, bronchospasm [17]. This is in line with Huseyin et al. [9] and $\mathrm{Hu}$ [24], who have clarified that NAC is a safe drug to the patients with acute viral hepatitis.

The role of NAC, a glutathione precursor, in the treatment of paracetamol-induced acute liver failure (ALF) is well established [25]. Also, in small trials, NAC has been used in nonparacetamol-induced ALF with variable results [26]. The clinical basis for the use of NAC is based on several mechanisms, of which the most important are: a) to facilitate the synthesis of depleted glutathione in AHA, and replenish hepatic stores of glutathione, b) it has vasodilating effects which improve microcirculatory blood flow and oxygen delivery to vital organs [27], c) increasing the blood flow by increasing the soluble nitric oxide activity in the gultamyl cyclase system, d) it acts as antioxidant that scavenges the free radicals [27], e) blocking oxidative stress and avoiding the accentuation of hepatic damage [28]. 
The data about using of oral NAC in the treatment of AHA are restricted. To our knowledge, there is no any study assessed the effects of NAC on AHA except one published study evaluating the effect of NAC on AVH (A $\&$ B), this article has shown that NAC did not have effect on the length of hospital stay of AVH infection and, on the period in which the ALT value came back to normal and also the prognosis of biochemical parameters [9].

\section{CONCLUSION}

This study reported that the use of oral NAC as adjunctive therapy for treatment of acute hepatitis A might be beneficial in decreasing the length of hospital stay. It is also reported that the use of NAC was safe because of absence of its side effects in these patients. There is an undoubted necessity for further research into the treatment of hepatitis A, and this study has identified a promising compound NAC, that may be an integral component of future HAV management.

\section{Funding: None.}

\section{Conflicts of interest: None.}

Ethical Approval: Approved.

\section{REFERENCES}

1. Ryan KJ, Ray CG (editors). Sherris Medical Microbiology (4th ed.), 2004; McGraw Hill. pp. 541-544.

2. Matheny, SC; Kingery, JE. "Hepatitis A." Am Fam Physician. 2012; 86 (11): 1027-34; quiz 1010-1012.

3. Brundage SC, Fitzpatrick AN. "Hepatitis A". Am Fam Physician. 2006; 73 (12): 2162-8.

4. "Hepatitis A Fact sheet $\mathrm{N}^{\circ} 328$ ". World Health Organization. July 2013. Retrieved 20 February 2014.

5. Global Burden of Disease Study 2013, Collaborators (22 August 2015). "Global, regional, and national incidence, prevalence, and years lived with disability for 301 acute and chronic diseases and injuries in 188 countries, 1990-2013: a systematic analysis for the Global Burden of Disease Study 2013.". Lancet (London, England). 386 (9995): 743-800.

6. Lozano, R. Global and regional mortality from 235 causes of death for 20 age groups in 1990 and 2010: a systematic analysis for the Global Burden of Disease Study 2010. Lancet, 2012. 380 (9859): 2095-2128.

7. Küçükardali Y, Cinan U, Acar HV, Ozkan S, Top C, Nalbant S, Cermik H, Cankir Z, Danaci M. Comparison of the therapeutic efficacy of 4- methylpyrazole and $\mathrm{N}$-acetylcysteine on acetaminophen (paracetamol) hepatotoxicity in rats. Curr Med Res Opin. 2002; 18:78-81.

8. Zhao C, Sheryl D, Zhou YX. Effects of combined use of diallyl disulfide and $\mathrm{N}$ acetyl-cysteine on acetaminophen hepatotoxicity in beta-naphthoflavone pretreated mice. World J Gastroenterol. 1998; 4:112-116.

9. Huseyin Gunduz, Oguz Karabay, Ali Tamer, Resat Özaras, Ali Mert, and Ömer Fehmi Tabak. $\mathrm{N}$-acetyl cysteine therapy in acute viral hepatitis. World J Gastroenterol. 2003 Dec 15; 9(12): 2698-2700.

10. Mandal AK, Sinha J, Mandal S, Mukhopadhyay $\mathrm{S}$, Das N. Targeting of liposomal flavonoid to liver in combating hepatocellular oxidative damage. Drug Deliv. 2002; 9:181-185.

11. Fischer J, Ganellin CR. Analogue-Based Drug Discovery. Weinheim: Wiley-VCH. 2006; p. 544.

12. "WHO Model List of Essential Medicines (19th List)" (PDF). World Health Organization. April 2015. Retrieved 8 December 2016.

13. Hansen NCG, Skriver A, Brorsen-Riis L, et al. Orally administered $\mathrm{N}$-acetylcysteine may improve general well-being in patients with mild chronic bronchitis. Respir Med. 1994;88:531-535

14. Riise GC, Larsson S, Larsson P, et al. The intrabronchial microbial flora in chronic bronchitis patients: a target for $\mathrm{N}$-acetylcysteine therapy? Eur Respir J. 1994;7:94-101

15. Walmsley SL, Khorasheh S, Singer J, et al. A randomized trial of $\mathrm{N}$-acetylcysteine for prevention of trimethoprim-sulfamethoxazole hypersensitivity reactions in Pneumocystis carinii pneumonia prophylaxis (CTN 057). Canadian HIV Trials Network 057 Study Group. J Acquir Immune Defic Syndr Hum Retrovirol. 1998; 19:498-505.

16. Look MP, Rockstroh JK, Rao GS, et al. Sodium selenite and $\mathrm{N}$-acetylcysteine in antiretroviral-naive HIV-1-infected patients: a randomized, controlled pilot study. Eur J Clin Invest. 1998; 28:389-397.

17. Akerlund B, Jarstrand C, Lindeke B, Sonnerborg A, Akerblad C, Rasool O. Effect of Nacetylcystine (NAC) treatment on HIV-1 infection: a double-blind placebo-controlled trial. Eur J Clin Pharmacol. 1996; 50:457-461.

18. Weiss L, Hildt E, Hofschneider PH. Antihepatitis B virus activity of $\mathrm{N}$ acetylcysteine, new aspect a well estabilished drug. Antiviral Res 1996; 32: 43-53

19. Neri S, Ierna D, Antoci S, Campanile E, D'Amico RA, Noto R. Association of alpha-interferon and acetyl cysteine in patients with chronic C hepatitis. Panminerva Med 2000; 423: 187-192

20. Beloqui O, Prieto J, Suarez M, Gil B, Qian CH, Garcia N, Civeira MP. N-acetyl cysteine enhances the response to interferon-alpha in chronic hepatitis C: a pilot study. J Interferon Res 1993; 13: 279-282 
21. Norberto Sotelo, Maria de Los Angeles, Alejandro Gonzalez, Nagasharmila Dhanakotti. Early treatment with $\mathrm{N}$-acetylcysteine in children with acute liver failure secondary to hepatitis A. Annals Hepatology 2009; 8 (4): 353-358.

22. Cochrean JB, Losek JD. Acute liver failure in children. Pediatr Emerg Care 2007; 23 (2): 129135.

23. Dart RC, Rumack BH. Patient tailored Nacetylcysteine administration. Ann Emerg Med 2007; 50 (3): 280-281.

24. Hu J, Zhang Q, Ren X, et al. Efficacy and safety of acetylcysteine in "non-acetaminophen" acute liver failure: A meta-analysis of prospective clinical trials. Clin Res Hepatol Gastroenterol. 2015; 39(5):594-599.

25. Smilkstein MJ, Bronstein AC, Linden C, Augenstein WL, Kulig KW, Rumack BH. Acetaminophen overdose: a 48-hour intravenous $\mathrm{N}$-acetylcysteine treatment protocol. Ann Emerg Med. 1991; 20(10):1058-1063.

26. Khalid Mumtaz, Zahid Azam, Saeed Hamid, Shahab Abid, Sadik Memon, Hasnain Ali Shah, and Wasim Jafri. Role of N-acetylcysteine in adults with non-acetaminophen-induced acute liver failure in a center without the facility of liver transplantation. Hepatol Int. 2009 Dec; 3(4): 563-570.

27. Ali Faisal Saleem, Qalab Abbas and Anwar ul Haque. Use of N-Acetylcysteine in Children with Fulminant Hepatic Failure Caused by Acute Viral Hepatitis. Journal of the College of Physicians and Surgeons Pakistan 2015, Vol. 25 (5): 354358.

28. Hu J, Zhang Q, Ren X, et al. Efficacy and safety of acetylcysteine in "non-acetaminophen" acute liver failure: A meta-analysis of prospective clinical trials. Clin Res Hepatol Gastroenterol. 2015; 39(5):594-599. 